\title{
Aceptabilidad del sabor de preparaciones hiposódicas con sustitutos de sal en pacientes diabéticos e hipertensos
}

\author{
Acceptability of the taste of low sodium \\ preparations with salt substitutes in \\ diabetic and hypertensive patients
}

\begin{abstract}
The purpose of this experimental study was to compare the acceptability of the taste between different preparations with low sodium in diabetic and hypertensive patients. It was conducted between April and May 2015 at the Centro de Atención Integral de Diabetes e Hipertensión (CEDHI) in Lima, Peru. Sixty-seven outpatient subjects from CEDHI were assessed; they were over 18 years of age, of both genders, diagnosed with hypertension or type 2 diabetes. In total 134 sensory tests were applied and 402 prepared food samples. The evaluated samples were rice, broth, chicken and cooked vegetables that were added with common salt, potassium chloride [25\%] with salt [75\%] and monosodium glutamate. In order to measure the results two hedonic tests were applied, ordered by preference and acceptability test (score of 1 to 5) were used. No significant global differences were found regarding the acceptability and preference in low sodium preparations. Therefore, the replacement of common salt by any of the substitutes is viable for patient's prescribed low-sodium diet (1500 mg Na / day).

Key words: low sodium diet; diabetes; hypertension; salt substitutes; acceptability.
\end{abstract}

\section{INTRODUCCIÓN}

La dieta es uno de los factores determinantes de la prevención, mejoramiento de la salud y calidad de vida de las personas (1). Los consumidores reportan que el gusto es un elemento decisivo e importante para la selección de los alimentos y que la variación en la percepción del sabor de la sal se asocia con diferencias en las respuestas hedónicas (grados de gusto o disgusto que se utilizan en las pruebas de aceptabilidad y preferencia) (2) a los alimentos altos en sodio y por lo tanto la ingesta de sodio (3).

El Perú muestra una evidente heterogeneidad en el perfil de la hipertensión y diabetes. En el 2015, se encontró que la prevalencia estandarizada por edad de la prehipertensión y la hipertensión fue $23,7 \%$ y $16,2 \%$ respectivamente y para el caso de prediabetes y diabetes mellitus fue $18.1 \%$ y $6.2 \%$ (4). La hipertensión es una comorbilidad frecuente de la diabetes mellitus y en el caso de la diabetes de tipo 2 es parte del
Angela Ayerbe-Azabache (1) Nancy Calderón-Ramírez (1) Marco Taboada (2) Percy Mayta-Tristán (3)

(1) Escuela de Nutrición, Universidad Peruana de Ciencias Aplicadas, Lima, Perú. (2) Centro de Atención Integral de Diabetes e Hipertensión, EsSalud, Lima, Perú. (3) Escuela de Medicina, Universidad Peruana de Ciencias Aplicadas, Lima, Perú.

Dirigir la correspondencia a: Angela Ayerbe-Azabache Jr. Victor Estremadoyro 181, San Borja, Lima, Perú Teléfono: (511) 3460961 Correo electrónico: angela_ayer@hotmail.com

Este trabajo fue recibido el 22 de Septiembre de 2015 aceptado con modificaciones el 1 de Marzo de 2016 y aceptado para ser publicado el 10 de Agosto de 2016. síndrome cardiometabólico, donde un factor importante es la sensibilidad a la sal (5).

Por esta razón, como prevención y tratamiento nutricional se prescribe una dieta hiposódica (6). Sin embargo, estas dietas presentan baja aceptabilidad en los pacientes. Esto se debe a que no perciben el sabor de las comidas por el bajo contenido de sal. La mayoría de pacientes con prescripción de dieta hiposódica no la cumplen, dificultando de esta manera el éxito del tratamiento nutricional (7).

Las sales neutrales como el cloruro de potasio $(\mathrm{KCl})$ son empleadas como un sustituto de la sal, sin embargo, en mayor concentración se puede percibir un sabor amargo (8) y metálico (9). Por otro lado, el glutamato monosódico (GMS) es un potenciador del sabor y contiene sólo un tercio de sodio presente en la sal común (10). El objetivo de este estudio fue comparar la aceptabilidad del sabor de preparaciones con bajo contenido de sodio usando glutamato monosódico, sal común 
y cloruro de potasio combinado con sal común en sujetos con prescripción de dieta hiposódica.

\section{SUJETOS Y MÉTODOS \\ Diseño de estudio}

Se realizó un estudio experimental en el Centro de Atención Integral de Diabetes e Hipertensión (CEDHI) de EsSalud ubicado en Lima-Perú durante el periodo de abril y mayo del 2015.

\section{Población}

Para estimar la muestra se realizó un estudio piloto en 16 personas, con ello se encontró el orden de preferencias y las medias en la prueba hedónica para cada producto evaluado. Se estimó la menor diferencia posible para uno de los productos y con estos datos se realizó el cálculo de tamaño de muestra. Se usó la fórmula para diferencias de medias relacionadas con una media de 3,42 $\pm 0,30$ para la muestra de sodio y de 3,7 \pm 0,47 para la muestra de glutamato monosódico con un nivel de significancia del $95 \%$ y una potencia de $80 \%$, se estimó un número mínimo de 60 personas que participen del estudio, considerando un $10 \%$ de pérdidas o rechazos, se debía enrolar a 66 personas, al final se logró incluir a 67 personas.

Los voluntarios fueron pacientes ambulatorios del CEDHI, mayores de 18 años de ambos géneros, diagnosticados de hipertensión arterial o diabetes tipo 2. Se excluyeron a personas que habían consumido alimentos o usado enjuague bucal una hora previa a la cata, a quienes presentaban lesiones en la boca (aftas, escorbuto, herpes) o sin dentadura. También se excluyó a fumadores, y a quienes estaban consumiendo algún fármaco adicional al tratamiento de hipertensión o diabetes, o si se encontraban con gripe o tomando fármacos para el tratamiento del resfrío.

\section{Preparación de muestras}

Se seleccionaron cuatro alimentos por su facilidad de preparación, aceptabilidad y consumo masivo; los cuales fueron: caldo (líquido), verduras cocidas (fibra), arroz blanco cocido (carbohidrato) y pollo cocido (proteína).

Nos basamos en el requerimiento diario de $2000 \mathrm{kcal}$ recomendado por la FDA (11), es decir se calculó el valor calórico total de cada preparación basada en una dieta de $2000 \mathrm{kcal} /$ día y con un máximo de 1500 mg/Na/día cantidad recomendada para una dieta hiposódica (12). Esta cantidad se adicionó individualmente con GMS, cloruro de sodio y una combinación de cloruro de potasio (25\%) con cloruro de sodio (75\%).

Para obtener la cantidad de cloruro de sodio a adicionar se calculó que si $1 \mathrm{~g}$ de $\mathrm{NaCl}$ contiene 400 mg de Na, 1500 mg/ $\mathrm{Na}$ /día es equivalente a $3.75 \mathrm{~g}$ de $\mathrm{NaCl}$ al día en una dieta de $2000 \mathrm{kcal} / \mathrm{dí}$. Con respecto a la mezcla de cloruro de potasio al 25\% se usó $5 \mathrm{~g}$ de muestra, aportando un total de $1500 \mathrm{mg}$ $\mathrm{Na}$. Por último, para obtener la cantidad de GMS, se calculó que si $1 \mathrm{~g}$ de GMS contiene $123 \mathrm{mg}$ de $\mathrm{Na}, 1500 \mathrm{mg} / \mathrm{Na} /$ día es equivalente a $12.2 \mathrm{~g}$ de GMS.

\section{Medición de variables}

Para evaluar la aceptabilidad se usó dos pruebas hedónicas, ambas validadas por el Instituto de Tecnólogos Alimentarios de EE.UU. (IFT). Las pruebas que utilizamos son prueba de preferencia por ordenamiento y prueba de aceptabilidad (2). Para esta última la valoración va de la categoría "me gusta mucho" a "me disgusta mucho", siendo la puntación del 1 al 5 respecto al sabor, donde el número mayor expresa el mayor agrado; mientras que para la prueba de ordenamiento la valoración va del 1 al 3, donde el número menor expresa mayor preferencia hacia la muestra escogida.

Cabe mencionar que para el adecuado orden de presentación de las muestras a los panelistas se consideró en el procedimiento seis órdenes diferentes en el que se degustaron las muestras, es decir seis posibles combinaciones que ayudó a no repetir una muestra más que la otra en el momento de la distribución (13) (tabla 1).

\section{Procedimientos}

Para la preparación de la muestra se usaron utensilios que no afecten abruptamente el sabor y aroma del alimento o preparación. Se utilizaron ollas de metal para la cocción de las diferentes preparaciones, utensilios de metal para pelar, picar o cortar los alimentos y tablas de picar de plástico para cada tipo alimento. Además, la preparación de las muestras se realizó un día previo a la cata, evitando olores fuertes el día de la evaluación.

De esta manera, el método de preparación normalmente se basa en documentos técnicos normalizados que lo regularizan por alimento o producto; sin embargo, de no existir estos se preparan de la forma en que habitualmente se consumen los alimentos (14). Para este estudio, se utilizó la misma cantidad de cada uno de los ingredientes, el mismo tiempo y temperatura de cocción; y la misma forma, tiempo y temperatura de conservación. Las cantidades de las muestras de alimentos sólidos fueron de 25 gramos y en alimentos líquidos como

\section{TABLA 1}

Orden de combinaciones de muestras.

Combinaciones

$\begin{array}{lc}1 & \mathrm{NaCl} \\ 2 & \mathrm{NaCl} \\ 3 & \mathrm{Na}+\mathrm{KCl} \\ 4 & \mathrm{Na}+\mathrm{KCl} \\ 5 & \mathrm{GMS} \\ 6 & \mathrm{GMS}\end{array}$

$\mathrm{NaCl}$ : cloruro de sodio.

$\mathrm{Na}+\mathrm{KCl}$ : cloruro de sodio $75 \%$ + cloruro de potasio $25 \%$.

GMS: glutamato monosódico.
Orden de muestras

$\begin{array}{cc}\mathrm{Na}+\mathrm{KCl} & \mathrm{GMS} \\ \mathrm{GMS} & \mathrm{Na}+\mathrm{KCl} \\ \mathrm{NaCl} & \mathrm{GMS} \\ \mathrm{GMS} & \mathrm{NaCl} \\ \mathrm{NaCl} & \mathrm{Na}+\mathrm{KCl} \\ \mathrm{Na}+\mathrm{KCl} & \mathrm{NaCl}\end{array}$


caldos, una cucharada equivalente a 15 mililitros (2).

Para la degustación de las muestras se utilizaron recipientes de plástico como indica la Norma Técnica Peruana (NTP) ISO 6658 (15), dado que este material no afecta al producto. Asimismo, la NTP indica que es necesario utilizar limpiadores de paladar de una muestra a otra y entre sesiones. Por ello, dado a la variedad de limpiadores de paladar, creímos conveniente utilizar agua sin gas y galleta de agua sin sal.

La codificación de la muestras fue con números y letras. De esta manera evitamos el uso de un solo digito numérico o alfabético que puede conllevar a errores. También las etiquetas utilizadas fueron de tres colores diferentes para identificar las muestras según el tipo de sustituto de sal usado. Las pruebas hedónicas tuvieron inicio a las 10A M de manera que los participantes tenían más de una hora sin haber comido después de su desayuno.

Por otro lado, el ambiente en donde se realizó el estudio fue en un consultorio del CEDHI, el cual fue adaptado para las finalidades del estudio (ventilación e iluminación adecuada). Éste se encontraba situado en las mismas instalaciones de los demás consultorios en donde se atienden los pacientes ambulatorios, por ello no existían ruidos que pudieran perturbar a los panelistas. Los participantes fueron entrando en grupos de dos personas, en el cual se realizaba la degustación de las muestras y el llenado de las pruebas sensoriales.

\section{Aspectos éticos}

El estudio fue revisado y aprobado por el Comité de Ética de la Red Rebagliati-CEDHI (545-OCID-RAR-EsSalud-2015) y por el comité de ética en la investigación (CEI) [CEI/374-0914] de la Facultad de Ciencias de la Salud de la Universidad Peruana de Ciencias Aplicadas (UPC).

Previo a la recolección de datos los participantes del estudio firmaron de forma voluntaria el consentimiento informado para poder participar en el estudio.
Análisis de datos

Los datos fueron ingresados por doble digitación al programa Excel, y luego del control de calidad exportados al programa STATA v11.0. Luego de verificar la normalidad en la distribución de los puntajes de la prueba hedónica con la prueba de Shapiro Wilk se expresaron con media y error estándar. Se evaluó las diferencias entre los puntajes con la prueba de ANOVA para muestras relacionadas tanto en la evaluación por sustituto de sal como por tipo de alimento. Para evaluar las diferencias en preferencias de sustituto de sal se usó la prueba de chi2. Se consideró un $\mathrm{p}<0,05$ como significativo.

\section{RESULTADOS}

Se incluyó a 67 participantes de los cuales 37 fueron mujeres. La edad osciló entre los 36 y 80 años, con una edad promedio de 65 años. La mayoría tenía antecedente de hipertensión y estaban en dieta hiposódica (tabla 2).

En relación a las pruebas sensoriales, cada participante probó seis muestras de dos de los cuatro grupos de alimentos, a los cuales se les adicionó sal común, cloruro de potasio con sal común y glutamato monosódico. En total fueron aplicadas 134 pruebas sensoriales y 402 muestras preparadas.

Cuando se evaluó las preferencias según el orden de los que más les gustaron, no se encontró diferencias tanto a nivel global como por cada alimento en particular entre ninguna de las preparaciones evaluadas $(p>0,05)$. Sin embargo, se aprecia una tendencia de preferencia de la combinación de sal con $\mathrm{KCl}$ al $25 \%$ para las preparaciones con pollo y de sal común para la de arroz (figura 1).

En las pruebas hedónicas no se encontraron diferencias en los puntajes obtenidos según tipo de alimento para cada sustituto ( $p>0,05)$, así como en las evaluaciones entre sustitutos para cada tipo de alimento o en forma global, excepto para la muestra de pollo, donde se evidenció que la combinación de sal con $\mathrm{KCl}$ al 25\% tuvo mayor aceptación que los otros

TABLA 2

Características de la población de estudio.

\begin{tabular}{lcc}
\hline Variables & $N$ & $(\%)$ \\
Género & & $(55,2)$ \\
Mujer & 37 & $(44,8)$ \\
Varón & 30 & $(11,1)$ \\
Edad (años)* & 65,1 & $(56,7)$ \\
Enfermedad & 38 & $(12,0)$ \\
Solo HTA & 8 & $(31,3)$ \\
Solo DM2 & 21 & $(27,3)$ \\
HTA y DM2 & 18 & $(88,1)$ \\
Obesidad & & $(43,3)$ \\
Régimen dietético & 59 & $(6,0)$ \\
Bajo en sal & 29 & $(1,5)$ \\
Hipocalórica & & \\
Hábitos de consumo de sal & 4 & \\
Adiciona sal a sus comidas & 1 & \\
Prefiere comida salada & & \\
\hline * Media y (desviación estándar). & & \\
DM2: diabetes mellitus tipo 2. & & \\
\hline
\end{tabular}


sustitutos $(p=0,024$, tabla 3$)$.

Adicionalmente, no se encontraron diferencias en las preferencias por sustituto según el orden en el que se administraron las muestras (tabla 1). Asimismo, no se encontraron diferencias según género y si la enfermedad de fondo era hipertensión ( $p>0,05)$. Sin embargo, las personas que estaban actualmente en dieta hiposódica tuvieron mayores puntajes en la prueba hedónica que los que no estaban en dieta hiposódica $(p<0,05)$, así como los que no tenían diabetes (tabla 4).

\section{DISCUSIÓN}

No encontramos diferencias significativas a nivel global de todos los alimentos evaluados en cuanto a la aceptabilidad y preferencia de las preparaciones con bajo contenido de sodio, tanto en las muestras con sal común y los sustitutos.

Se ha demostrado que es posible disminuir en 30\% el sodio en platos complejos sin afectar la aceptabilidad (16). Asimismo, en un estudio realizado en Lima se observó que con una reducción de sodio al $20 \%$ en panes no se aprecian cambios en el gusto (17). Igualmente, en el Reino Unido se realizó un estudio en donde se redujo gradualmente el contenido de sodio hasta $25 \%$ por un período de seis semanas, sin ser percibido por los consumidores (18). En el presente estudio la reducción fue de $37,5 \%$ de la cantidad de sodio recomendada en personas sanas ( $2400 \mathrm{mgNa} /$ día), sin que los sujetos de estudio detectaran esta disminución. Es importante

\section{FIGURA 1}

Preferencias por ordenamiento según alimento y aditivo de preparaciones hiposódicas en pacientes con diabetes mellitus tipo 2 o hipertensión arterial.

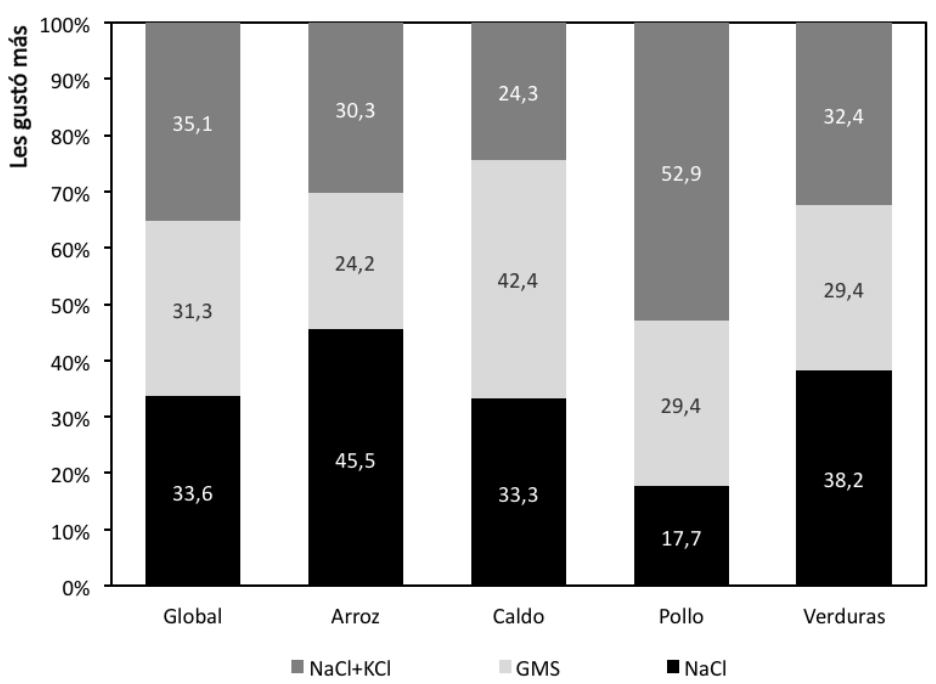

Se puede apreciar diferencias en las preferencias en las preparaciones con el producto proteico (pollo)

$\mathrm{NaCl}$ : cloruro de sodio.

$\mathrm{Na}+\mathrm{KCl}$ : cloruro de sodio $75 \%+$ cloruro de potasio $25 \%$

GMS: glutamato monosódico.

\section{TABLA 3}

Comparación por alimento y por aditivo de la prueba de aceptabilidad para pacientes con diabetes tipo 2 o hipertensión arterial.

\begin{tabular}{|c|c|c|c|c|c|c|c|}
\hline \multirow[t]{2}{*}{ Alimento } & \multicolumn{2}{|c|}{ Sal común } & \multicolumn{2}{|c|}{ Sal con $\mathrm{KCl}$} & \multicolumn{2}{|c|}{ Glutamato monosódico } & \multirow[t]{2}{*}{$\mathrm{p}$} \\
\hline & Media & (EE) & Media & (EE) & Media & (EE) & \\
\hline Arroz & 3,58 & $(0,16)$ & 3,30 & $(0,19)$ & 3,12 & $(0,22)$ & 0,242 \\
\hline Caldo & 3,39 & $(0,14)$ & 3,30 & $(0,16)$ & 3,21 & $(0,18)$ & 0,594 \\
\hline Pollo & 3,09 & $(0,15)$ & 3,71 & $(0,17)$ & 3,32 & $(0,17)$ & 0,024 \\
\hline Verduras & 3,44 & $(0,18)$ & 3,29 & $(0,20)$ & 3,35 & $(0,12)$ & 0,684 \\
\hline Global & 3,37 & $(0,08)$ & 3,40 & $(0,09)$ & 3,25 & $(0,09)$ & 0,648 \\
\hline $\mathrm{p}$ & \multicolumn{2}{|c|}{0,174} & \multicolumn{2}{|c|}{0,085} & \multicolumn{2}{|c|}{0,487} & \\
\hline
\end{tabular}

EE: error estándar. 
resaltar que tan sólo reduciendo $15 \%$ de sodio se pueden prevenir 13.8 millones de muertes por enfermedades crónicas no transmisibles (19).

Por otro lado, el cloruro de potasio combinado con cloruro de sodio resultó tener más aceptabilidad con el producto cárnico, y similar con los otros productos, situación que se ha demostrado en estudios previos que lo han presentado como un sustituto de sal (20). Se ha demostrado una actitud positiva de los consumidores frente a productos cárnicos donde el sodio estaba reducido, pero combinado con cloruro de potasio mostraron no solo un comportamiento intencional de consumir estos productos sino también les agradó de manera similar que los productos cárnicos convencionales (21). Asimismo, en filetes de tilapia, se menciona que en concentraciones de cloruro de potasio entre $20-40 \%$ no influyen significativamente en el sabor del producto y que el cloruro de potasio es comúnmente utilizado como sustituto de productos cárnicos (22). Por lo que se reafirma que existe mejor aceptabilidad en productos cárnicos que son adicionados con sales con cloruro de potasio.

El estudio presenta algunas limitaciones al no escogerse a los panelistas dentro de un rango de edad homogéneo, ya que la edad hace variar la capacidad del individuo de percibir sabores, sin embargo, la correlación entre los puntajes de las pruebas y la edad fueron menores de 0,1 todos ellos con valores de $p>0,4$, por lo que la edad no influyó en nuestros resultados al ser la mayoría de participantes de un rango homogéneo. Por otro lado, se considera que el etiquetado de las muestras puede ser una fuente de sesgo, ya que los colores pudieron ayudar al panelista a reconocer ciertos atributos de las muestras. También, al ser pacientes diabéticos e hipertensos, casi todos consumían al menos un medicamento que se conoce que puede alterar el sabor, como los diuréticos, bloqueadores de canal de calcio, inhibidor de la enzima convertidora de angiotensina o hipoglicemiantes (23); sin embargo al ser la población objetivo del estudio no había forma de controlar este aspecto, además que es más cercano a lo que sucede en la realidad. Por último, el número de sujetos que no estaban en actual consumo de dieta hiposódica no permitió hacer un análisis diferenciado. Futuros estudios podrían evaluar la aceptabilidad de estos sustitutos en sujetos sin dieta hiposódica, ya que la baja aceptación de las preparaciones se puede deber no al sustituto sino a la preparación hiposódica en sí.

En un principio se pensó que el glutamato monosódico iba a ser el sustituto de sal con mayor aceptabilidad. Luego de los resultados se observó que el sustituto de cloruro de potasio con sal común resulta ser una buena opción para reducir la ingesta de sodio en personas con prescripción de dieta hiposódica. Una de las razones es que el glutamato monosódico tiene un sabor muy característico que tal vez no sea del agrado de todos, mientras que las sales de sodio combinadas con cloruro de potasio, en donde el porcentaje de potasio no sea mayor al $25 \%$, las personas no perciben un cambio en el sabor ni el sabor metálico propio del cloruro de potasio que se evidencia en mayores cantidades a esta. Asimismo, en un estudio se utilizaron concentraciones con $25 \%$, 33\% y $55 \%$ de cloruro de potasio combinado con sal común que fueron comparadas con un control de cloruro de sodio al 100\%. Los resultados fueron que en concentraciones de $25 \%$ no hubo diferencia en el sabor, sin embargo en concentraciones de $33 \%$ y $55 \%$ se distinguieron diferencias (24). Por otro lado, resulta ser más práctico en el día a día de los consumidores, ya que el volumen de sal sería similar al que emplean diariamente en la preparación de sus alimentos, mientras que en el caso del glutamato monosódico la cantidad que se tendría que adicionar sería de mayor volumen. Con respecto a la cantidad de potasio que se estaría consumiendo por día sólo representa $36 \%$ del requerimiento diario recomendado por la OMS (Organización Mundial de la Salud, 2012) (25) que es $3.5 \mathrm{~g}$ de potasio por día. Sin embargo, hay que tener en cuenta, que este sustituto no está recomendado en pacientes que tengan enfermedad renal crónica terminal, a la cual pueden llegar muchos pacientes diabéticos e hipertensos.

\section{CONCLUSIÓN}

Todos los alimentos con sus respectivos aditivos fueron aceptados en cuanto a su sabor, ya que las diferencias encontradas fueron mínimas, yendo de una puntuación del sabor que

\section{TABLA 4}

Diferencias en puntaje de pruebas de aceptabilidad según características de los participantes ( $\mathrm{n}=402$ pruebas).

\begin{tabular}{llll}
\hline Variable & Puntaje & $(\mathrm{EE})$ & $p$ \\
& Media & & 0,035 \\
Diabetes & & $(0,07)$ & $(0,07)$ \\
Sí & 3,22 & 0,315 \\
No & 3,43 & $(0,05)$ & 0,057 \\
Hipertensión & 3,36 & $(0,13)$ & 0,001 \\
Sí & 3,21 & $(0,07)$ & $(0,08)$ \\
No & 3,43 & $(0,05)$ & 0,110 \\
Género & 3,24 & $(0,14)$ & $(0,07)$ \\
Mujer & 3,40 & $(0,07)$ & \\
Varón & 2,92 & &
\end{tabular}

EE: error estándar. 
va de indiferente a agradable. Por ello, es posible el reemplazo de la sal común por cualquiera de los aditivos, ya que no existen grandes diferencias en cuanto al sabor de los sustitutos de sal y la sal común. Asimismo, el glutamato monosódico pese a ser un aditivo reconocido por su característica de ser un gran potenciador del sabor, no mostró marcar ninguna diferencia en el estudio respecto a los otros aditivos, ya que los puntajes se mantienen similares para cada uno de ellos. Finalmente, la sustitución en un $25 \%$ con cloruro de potasio en la muestra proteica evidenció mayor aceptación en relación al glutamato monosódico y la sal común.

\section{RESUMEN}

El objetivo de este estudio experimental fue comparar la aceptabilidad del sabor entre diferentes preparaciones con bajo contenido de sodio en pacientes diabéticos e hipertensos. Se realizó entre abril y mayo del 2015 en el Centro de Atención Integral de Diabetes e Hipertensión (CEDHI) en Lima, Perú. Las personas evaluadas fueron 67 pacientes ambulatorios del CEDHI, mayores de 18 años de ambos géneros, diagnosticados de hipertensión arterial o diabetes tipo 2 . En total fueron aplicadas 134 pruebas sensoriales y 402 muestras preparadas. Las preparaciones que fueron sometidas a evaluación fueron arroz, caldo, pollo y verduras cocidas, adicionándoles sal común, cloruro de potasio [25\%] con sal común [75\%] y glutamato monosódico. Para medir los resultados se utilizaron dos pruebas hedónicas, de preferencia por ordenamiento y prueba de aceptabilidad (puntuación del 1 al 5). En los resultados no se encontraron diferencias significativas a nivel global en cuanto a la aceptabilidad y preferencia de las preparaciones con bajo contenido de sodio. Por lo cual, el reemplazo de la sal común por cualquiera de los sustitutos es viable para pacientes con prescripción de dieta hiposódica (1500 mg Na / día).

Palabras clave: dieta hiposódica; diabetes; hipertensión; sustitutos de sal; aceptabilidad.

Conflictos de interés: Los autores declaran no tener conflictos de interés con la publicación de este artículo

Fuente de financiamiento: Autofinanciado.

\section{BIBLIOGRAFÍA}

1. Nakasato $M$, Strunk $C, G$ Guimarães Rezende $M$, Bocchi $E$. Does the diet with low sodium level is indeed a recommendation that helps all patients with stable heart failure? Arq Bras Cardiol 2010; 94 (1): 87-95

2. Hernandez E. Sensory evaluation. Bogotá: National Media Center for learning; 2005

3. Hayes JE, Sullivan BS, Duffy VB. Explaining variability in sodium intake through oral sensory phenotype, salt sensation and liking. Physiol Behav. 2010;100(4):369-80.

4. Bernabé-Ortiz A, Carrillo-Larco RM, Gilman RH, Checkley W, Smeeth L, Miranda JJ; CRONICAS Cohort Study Group. Contribution of modifiable risk factors for hypertension and type-2 diabetes in Peruvian resource-limited settings. J Epidemiol Community Health. 2016;70(1):49-55.

5. Aaron $K J$, Sanders PW. Role of dietary salt and potassium intake in cardiovascular health and disease: a review of the evidence. Mayo Clin Proc. 2013;88(9):987-95.

6. Suckling RJ, He FJ, Macgregor GA. Altered dietary salt intake for preventing and treating diabetic kidney disease. Cochrane Database Syst Rev. 2010;8(12):CD006763.

7. Gorosquieta AM, Azanza DB, Fernandez AC, MG Madrazo,
Hernando Vicente A. Perception of hypertensive patients about the low sodium diet. Enferm Cardiol. 2008; 44 (2): 15-20.

8. Hoogue SE. Impact of potassium chloride on saltiness, bitterness, and other sensory characteristics in model soup systems. [Tesis] Kansas: Kansas State University; 2008.

9. Li N, Prescott J, Wu Y, Barzi F, Yu X, Zhao L, et al. The effects of a reduced-sodium, high-potassium salt substitute on food taste and acceptability in rural northern China. $\mathrm{Br}$ J Nutr. 2009;101(7):1088-93.

10. Jinap S, Hajeb P. Glutamate. Its applications in food and contribution to health. Appetite. 2010;55(1):1-10.

11. US Department of Health and Human Services, US Department of Agriculture. Dietary guidelines for Americans 2005. 6th ed. Washington, DC: US Department of Health and Human Services, US Department of Agriculture; 2005.

12. National Institutes of Health [Website] US: Dietary supplement label date basis; c51c896aa158. [Updated: February 2014; Accessed April 26, 2014]. Available in: http://www. ds/d.n/m.nih.gov/ds/d/dailyvalue.jsp

13. Mason RL, Nottingham SM. Sensory evaluation manual. Brisbane: University of Queensland; 2002.

14. Ibañez F, Barcina Y. Sensory analysis of food: methods and applications. Barcelona: Springer; 2001.

15. INDECOPI. Peruvian Technical Standard NTP-ISO 6658:2008 Sensory analysis. Methodology. General guidelines. Lima: INDECOPI; 2008.

16. Mariloux M, Walsh CM, Van Der Merwe CA. Consumer acceptability and salt perception of food with a reduced sodium content. J Fam Ecol Consum Sci. 2003; 31:12-20.

17. Saavedra-Garcia L, Sosa-Zevallos V, Diez-Canseco F, Miranda JJ, Bernabe-Ortiz A. Reducing salt in bread: a quasi-experimental feasibility study in a bakery in Lima, Peru. Public Health Nutr. 2016;19(6):976-82.

18. Girgis S, Neal B, Prescott J, Prendergast J, Dumbrell $S$, et al. A one-quarter reduction in the salt content of bread can be made without detection. Eur J Clin Nutr. 2003;57(4):616-20.

19. Asaria P, Chisholm D, Mathers C, Ezzati M, Beaglehole R. Chronic disease prevention: health effects and financial costs of strategies to reduce salt intake and control tobacco use. Lancet. 2007; 370(9604):2044-53.

20. Ainsworth P, Piper B, Knott S. Consumer acceptability of foods using low salt levels and salt substitutes. J Consum Stud Home Econ. 1993;17(4):305-11.

21. Guardia MD, Guerrero L, Gelabert J, Gou P, Arnau J. Consumer attitude towards sodium reduction in meat products and acceptability of fermented sausages with reduced sodium content. Meat Sci. 2006;73(3):484-90.

22. Monteiro ML, Mársico ET, Canto AC, Costa-Lima BR, Lázaro CA, Cruz AG, et al. Partial sodium replacement in tilapia steak without loss o acceptability. Food Sci Technol Int. 2015;21(4):295-305.

23. Sánchez $K L$, Rodríguez $L R$, Sosa RS, Green DR. Taste disorders in oncology patients. Gac Med Mex. 2009; 8(5):211-15.

24. Saavedra-Garcia L, Bernabe-Ortiz A, Gilman RH, DiezCanseco F, Cárdenas MK, Sacksteder KA, Miranda JJ. Applying the triangle taste test to assess differences between low sodium salts and common salt: evidence from Peru. PLoS One. 2015;10(7):e0134700.

25. World Health Organization (WHO). Guideline: Potassium intake for adults and children. Geneva: WHO; 2012. 